\title{
Fatores de Variação e Parâmetros Genéticos dos Períodos de Serviço e Seco em Bovinos da Raça Holandês no Estado de Minas Gerais ${ }^{1}$
}

\author{
Idalmo Garcia Pereira², Tarcísio de Moraes Gonçalves ${ }^{3}$, Antonio Ilson Gomes de Oliveira4, \\ Nilson Milagres Teixeira ${ }^{5}$
}

\begin{abstract}
RESUMO - Objetivou-se com o presente estudo avaliar o efeito de alguns fatores de meio sobre as características período de serviço (PSER) e período seco (PS), bem como estimar parâmetros genéticos para estas características, em vacas holandesas. Foram analisados 6.485 registros de PSER e PS e consideraram-se no modelo os efeitos fixos de núcleo de criadores, ano de parto, estação de parto, nível de produção (NP), grupo genético (GG), idade ao parto em classe (IDC), interações de primeira ordem entre NP, GG e IDC e efeito aleatório de touro. As médias, os erros-padrão e os coeficientes de variação para o PSER e PS foram 121,21 (0,69) dias e 46,10\% e 92,27 (0,59) dias e $51,71 \%$, respectivamente. Constataram-se efeitos de touro, núcleo, ano, estação e nível de produção e interação GG com NP sobre PSER. Para PS, observaram-se efeitos de núcleo, ano, estação, nível de produção, idade ao parto em classes, interação GG com NP e de touro. Constatou-se não haver influência de GG sobre as características estudadas e redução nas médias ajustadas do PSER e PS com o passar dos anos. Estimativas de herdabilidade em análises uni e bivariada foram de 0,052 e 0,027 para PSER e 0,004 e 0,0001 para PS, respectivamente, e de repetibilidade, 0,06 e 0,03 , respectivamente, indicando que ambas as características são basicamente influenciadas por efeitos de meio ambiente. Correlações genética, fenotípica e de ambiente de 0,55; 0,72; e 0,73, respectivamente, entre PSER e PS, indicam que vacas com períodos de serviço de maior duração têm maiores períodos secos.
\end{abstract}

Palavras-chave: período de serviço, período seco, parâmetros genéticos, raça Holandês

\section{Source of Variation and Genetic Parameters of Days Open and Days Dry in Holstein Dairy Cattle in Minas Gerais State}

\begin{abstract}
The objective of this work was to evaluate the effect of some environmental factors on days open (DO) and days dry (DD), as well as to estimate genetic parameters for these traits in Holstein breed cows. A total of 6,485 records of DO and DD were used. The database were analyzed using a model that included the fixed effects of dairy group farmers, calving year, calving season, production level (PL), genetic group (GG), calving age class (CA) and the first order interactions between PL, GG and CA, and the random effect of bulls. The means, standard error and coefficients of variation for DO and DD were $121.21(0.69)$ days, 46.10\% and 92.27(0.59) days, $51.71 \%$, respectively. For DO, effects of bull, dairy group farmers, calving year, calving season, PL were significant and was observed interaction between PL and GG. For DD, the effects of dairy group farmers, calving year, calving season, PL, CA and the interaction between PL and GG and for bull were significant. Genetic group did not affected the studied variables and a reduction in DO and DD with year was observed. The heritability estimate in univariate and bivariate analyses were 0.052 and 0.027 for DO, and 0.004 and 0.0001 for DD, respectively. The repeatabilities for DO and DD were 0.06 and 0.03 , respectively. It was observed that the environmental effects are the most important on these traits. The genetic, phenotypic and environmental correlations between DO and DD $(0.55,0.72$ and 0.73 , respectively) show that cows with higher DO had greater days dry.
\end{abstract}

Key Words: days open, days dry, genetic parameters, Holstein breed

\section{Introdução}

A pecuária leiteira é uma das principais atividades do setor agropecuário brasileiro e, atualmente, apesar de apresentar expressiva produção de leite, ainda encontra-se com índices produtivos e reprodutivos aquém dos almejados para uma pecuária desenvolvida.
O desempenho reprodutivo de uma população de bovinos é fator determinante do progresso genético anual, apresentando relação negativa com a produção de leite. Este pode ser ainda mais intenso em vacas da raça Holandês selecionadas para alta produção de leite em regiões tropicais e subtropicais, devido ao estresse climático, com prejuízo para o progresso

\footnotetext{
${ }^{1}$ Parte da Dissertação de Mestrado do primeiro autor, apresentada ao DZO da Universidade Federal de Lavras.

2 Professor Visitante do DZO da UFLA.

3 Professor Assistente do DZO da UFLA. E.mail: tarcisio@ufla.br

${ }^{4}$ Professor Titular Aposentado do DZO da UFLA e bolsista do CNPq - Lavras - MG. E.mail: ailson@ ufla.br

5 Pesquisador do CNPGL - EMBRAPA e bolsista do CNPq - Juiz de Fora - MG.
} 
genético das características produtivas, pelo pior desempenho reprodutivo.

O período de serviço (PSER) é um dos principais indicadores de eficiência reprodutiva, pois alterações neste parâmetro influenciam diretamente o intervalo de partos. Vários fatores têm sido relacionados nos estudos envolvendo o PSER, entre eles a idade da vaca e/ou ordem de parto (HILLERS et al., 1984), a estação de parto (MARTI e FUNK, 1994), o grupo genético (BUTTE e DESHPANDE, 1987), o ano de parto e a região onde está localizado o rebanho. Adicionalmente, tem sido considerado o efeito de nível de produção do animal (NEBEL e McGILLIARD, 1993).

O período seco (PS) tem também grande influência sobre a produção de leite. A condição física da vaca ao parto reflete diretamente no desempenho produtivo, necessitando assim de um período de descanso entre duas lactações subseqüentes para permitir a regeneração das células do tecido secretor da glândula mamaria e acúmulo de reservas corporais para a próxima lactação (DIAS e ALLAIRE, 1982; RIBAS et al., 1984).

Os fatores considerados em estudos envolvendo o PSER também são comuns para PS: idade da vaca e/ou ordem de parto, estação do parto e nível de produção (SCHAEFFER e HENDERSON, 1972). Informações sobre grupo genético são restritas, tendo em vista que, nos países de origem da maioria dos trabalhos, não há preocupação quanto a este efeito, pois os animais para produção de leite são de raças "puras".

Embora a variabilidade genética para o PSER e o PS seja bastante estudada em países desenvolvidos, no Brasil, as pesquisas a respeito são incipientes. Estimativas baixas de herdabilidade $\left(\hat{\mathrm{h}}^{2}\right)$ para o PSER: 0,01; 0,05; e 0,09 na primeira, segunda e lactações seguintes no trabalho de SMITH e LEGATES (1962), na raça Holandês; 0,$03 ; 0,04$; e 0,05 na primeira, segunda e terceira lactações, respectivamente, por RAHEJA et al. (1989) no Canadá; e 0,045 em estudos mais recentes, MARTI E FUNK (1994), utilizando REML, sob modelo animal, evidenciaram baixa influência genética para esta característica.

SCHAEFFER e HENDERSON (1972), ao obterem estimativas de $\mathrm{h}^{2}$ de até 0,34 para o PS, especulam que esta característica poderia apresentar alguma base genética. Entretanto, valores de 0,062 encontrados por FUNK et al. (1987) sugerem que esta característica é muito mais resultado do manejo e outras influências ambientais. No Brasil, VALENTE et al.
(1994) e VALENTE et al. (1995), utilizando o método REML e o procedimento livre de derivadas (DF), obtiveram $\hat{h}^{2}$ de 0,12 , diminuindo para 0,08 , quando a produção total na lactação foi incluída na análise como covariável.

Os valores muitos baixos $(<0,10)$ para as estimativas de repetibilidade obtidos por MAKUZA e McDANIEL (1996) evidenciam que os efeitos permanentes de ambiente são de menor importância para estas características.

Em geral, os valores para correlação genética entre PSER e PS são baixos, com exceção dos obtidos por MOORE et al. (1990), com vacas das raças Ayrshire e Holandesa, que obtiveram 0,44 e 0,48 , respectivamente. Recentemente, MAKUZA e McDANIEL (1996), com vacas da raça Holandês, obtiveram valores para a primeira e segunda lactações de $-0,16$ e $-0,54$, respectivamente.

Diferenças genéticas entre as populações, metodologias de análise, utilização de modelos diferentes e variação no número de registros e confiabilidade dos dados utilizados são enumeradas como causas mais prováveis para explicar as variações encontradas entre os parâmetros genéticos (SILVA, 1995).

O objetivo do presente estudo foi avaliar o efeito de algumas causas de variação de período de serviço e seco e estimar parâmetros genéticos destas características em vacas da raça Holandês, criadas no Estado de Minas Gerais.

\section{Material e Métodos}

Os dados foram obtidos junto ao Serviço de Controle Leiteiro - SCL da Associação dos Criadores de Gado Holandês de Minas Gerais (ACGHMG). Inicialmente, o arquivo de dados possuía 49.276 registros de lactações de 25.458 vacas, pertencentes a 334 rebanhos, distribuídos em seis núcleos de criadores, com partos observados entre 1971 e 1995. Foram eliminados os registros: anteriores a 1990; com duração da lactação inferior a 150 e superior a 450 dias; com produção inferior a $1.000 \mathrm{~kg}$ de leite; com idades ao parto inferiores a 20 e superior a 200 meses; com período de serviço inferior a 20 e superior a 300 dias; e com período seco superior a 300 dias.

Após esta etapa, foram feitas outras eliminações, como vacas que não apresentavam a primeira ordem de parto e vacas acima da quinta ordem de parto, devido ao pequeno número de registros. Finalmente foi considerado um mínimo de duas filhas por pai e que cada subclasse RAE (rebanho-ano-estação) conti- 
vesse pelo menos quatro observações. Para as análises de PSER e PS, o novo arquivo passou a constituir-se de 6485 lactações de 4068 vacas, filhas de 359 touros, provenientes de 134 rebanhos, distribuídos nos anos de 1990 a 1994. A preparação dos dados e as análises estatísticas foram feitas utilizando-se o Statistical Analysis System (STATISTICAL ANALYSIS SYSTEM - SAS, 1996).

Foram avaliados os efeitos das seguintes causas de variação: estação de parição (águas, de outubro a março e seca, de abril a setembro); grupo genético (31/32 Holandês-Zebu, Puro por cruza com geração controlada e Puro de origem), 15 classes de idade ao parto (20-25, 26-27, 28-29, 30-31, 32-35, 36-39, 40$43,44-47,48-52,53-57,58-62,63-68,69-74,75-82 \mathrm{e}$ $\geq 83$ meses) e nível de produção na lactação (baixo, médio e alto). Para se determinarem os níveis de produção de leite, estimaram-se a média $(5635 \mathrm{~kg})$ e o desvio-padrão (1835 kg); a seguir definiu-se como nível baixo todas as lactações com menos de $3800 \mathrm{~kg}$ de leite (um desvio-padrão abaixo da média), como nível médio as lactações entre 3800 e 7470 kg de leite (um desvio-padrão abaixo e outro acima da média) e como nível alto as lactações acima de $7470 \mathrm{~kg}$ (um desvio-padrão acima da média).

Para estudar as causas de variação que influenciam os períodos de serviço e período seco, o modelo utilizado levou em consideração os efeitos fixos de núcleo, ano de parto, estação de parto, grupo genético, nível de produção na lactação, classe de idade da vaca ao parto e interações de primeira ordem de grupo genético com nível de produção, grupo genético com classe de idade e nível de produção com classe de idade, além dos efeitos aleatórios de reprodutor e erro.

Para a estimação dos parâmetros genéticos, foi utilizado o programa MTDFREML (Multiple-Trait Derivative Free Restricted Maximum Likelihood), sendo que nos dois tipos de análises efetuadas, univariada e bivariada, estabeleceu-se como critério de convergência a variância do SIMPLEX menor que $10^{-9}$.

Em análise univariada, o modelo linear misto utilizado em sua forma matricial foi:

$$
y=X \underset{\sim}{\beta}+Z \underset{\sim}{a}+W \underset{\sim}{c}+\underset{\sim}{e}
$$

em que

$y$
$\sim$

período de serviço ou período seco;

$X=$ matriz de incidência dos efeitos fixos; $\begin{aligned} & \beta \\ & \sim\end{aligned}=$ vetor de efeitos fixos (rebanho-ano-estação, grupo genético, nível de produção e idade da vaca ao parto em classe);

$Z$ = matriz de incidência dos efeitos aleatórios (efeitos genéticos diretos);

$\stackrel{a}{\sim}=$ vetor dos efeitos aleatórios dos valores genéticos diretos do animal (efeito animal);

$W=$ matriz de incidência dos efeitos aleatórios permanentes de ambiente;

$\stackrel{c}{\sim}=$ vetor de efeitos permanentes de ambiente; $\mathrm{e}$

${ }^{e}=$ vetor dos erros associados a cada observação.

Em análise bivariada, utilizou-se o mesmo modelo proposto anteriormente, porém bivariado. De maneira geral, o modelo pode ser assim representado:

$$
\begin{gathered}
\underset{\sim}{y_{1}}=X_{1} \underset{\sim}{\beta_{1}}+Z_{1} \underset{\sim}{a_{1}}+W_{1} \underset{\sim}{c_{1}}+\underset{\sim}{e_{1}} \\
\underset{\sim}{y_{2}}=X_{2} \underset{\sim}{\beta_{2}}+Z_{2} \underset{\sim}{a_{2}}+W_{2} \underset{\sim}{c_{2}}+e_{\sim}^{e_{2}}
\end{gathered}
$$

em termos matriciais, este modelo seria:

$$
\left[\begin{array}{c}
y_{1} \\
\tilde{y_{2}} \\
\sim
\end{array}\right]=\left[\begin{array}{cc}
X & 0 \\
0 & X
\end{array}\right]\left[\begin{array}{c}
\beta_{1} \\
\tilde{\beta}_{2} \\
\sim
\end{array}\right]+\left[\begin{array}{cc}
Z & 0 \\
0 & Z
\end{array}\right]\left[\begin{array}{c}
a_{1} \\
\tilde{a_{2}} \\
\sim
\end{array}\right]+\left[\begin{array}{cc}
W & 0 \\
0 & W
\end{array}\right]\left[\begin{array}{c}
c_{1} \\
\tilde{c_{2}} \\
\sim
\end{array}\right]+\left[\begin{array}{c}
e_{1} \\
\tilde{e_{2}} \\
\sim
\end{array}\right]
$$

em que o índice 1 representa a característica período de serviço e o índice 2, o período seco.

\section{Resultados e Discussão}

\section{Fatores de variação}

A média e respectivo erro-padrão de 6485 períodos de serviço foram de 121,21 $(0,69)$ dias, com coeficiente de variação de $46,10 \%$. Esta média está acima da encontrada na literatura para que se tenha ótimo desempenho reprodutivo (cerca de 60 dias), possibilitando intervalo de partos em torno de 13 a 14 meses, o qual, embora possa parecer elevado para gado Holandês bem manejado, pode ser considerado aceitável para as condições tropicais. No entanto, melhorias no manejo pré e pós-parto, que permitam melhor condição corporal no pré-parto, acompanhada de assistência sanitária mais criteriosa, bom manejo nutricional, apurada detecção de cio e, finalmente, uso correto das técnicas de inseminação, podem conjuntamente contribuir para redução do PSER.

Na literatura, em sua maioria, as médias de PSER são muito variáveis com valores médios superiores 
(DIAS et al., 1985; SILVA et al., 1992; CAMPOS et al., 1994; CASTRO et al., 1995; e MAKUZA e McDANIEL, 1996) ou inferiores (RIBAS et al., 1995) ao estimado no presente trabalho.

A média e respectivo erro-padrão do período seco foram de 92,3 $(0,59)$ dias, com coeficiente de variação de $51,71 \%$. Esta média é relativamente alta, pois a maior parte dos autores recomenda um PS de 60 dias, para se maximizar a produção na vida útil da vaca. Esta média elevada pode ser reflexo do valor, também alto, obtido para PSER. Poucos autores têm obtido médias superiores à deste estudo, entre eles KHATTAB e ASHMAWY (1988) e TEIXEIRA et al. (1995). O alto CV encontrado pode ser indicativo da considerável interferência do produtor a que esta característica está sujeita.

Pela análise de variância (Tabela 1), observamse para PSER efeitos significativos de touro, núcleo, ano, estação e nível de produção $(\mathrm{P}<0,01)$ e da interação de grupo genético com nível de produção $(\mathrm{P}<0,08)$, e para PS, efeitos significativos de núcleo, ano, estação e nível de produção, idade da vaca em classes e da interação de grupo genético com nível de produção $(\mathrm{P}<0,01)$ e de touro $(\mathrm{P}<0,07)$.
Com relação ao efeito dos núcleos para PSER e PS (Tabela 2), as variações que ocorrem entre as médias, no geral, vêm a confirmar que as diferenças regionais, como solo, clima, manejo, nutrição e questões culturais inerentes aos criadores de cada núcleo, podem afetar o desempenho reprodutivo dos animais, sendo que, no caso do PS, acrescenta-se o fato desta característica ser fortemente influenciada pelo manejo adotado pelo criador, que, muitas vezes, seca a vaca para que possa recuperar suas reservas corporais e, também, ocorrer a regeneração do tecido secretor da glândula mamaria para a lactação subseqüente, ou até mesmo recuperar de alguma enfermidade, o que é menos freqüente.

Oscilações ocorridas para PSER e PS entre os anos de parição (Tabela 3) são, provavelmente, resultantes das diferenças nos manejos nutricional, reprodutivo e sanitário. Podem ser também atribuídas às mudanças da freqüência gênica na população, seja por seleção, migração ou emigração de genes, conseguida às custas da compra e venda de reprodutores. Reduções no PSER e no PS, no último ano, podem ser resultado de evolução não só das técnicas de manejo, como também da genética para

Tabela 1 - Análise de variância dos períodos de serviço e seco de vacas da raça Holandês Table 1 - Analysis of variance of days open and days dry of Holstein breed cows

\begin{tabular}{|c|c|c|c|c|c|c|c|}
\hline \multirow[b]{2}{*}{$\begin{array}{l}\text { Fonte de variação } \\
\text { Source of variation }\end{array}$} & \multirow[b]{2}{*}{$\begin{array}{l}\text { G } \\
D F\end{array}$} & \multicolumn{3}{|c|}{$\begin{array}{c}\text { Período de serviço } \\
\text { Days open } \\
\end{array}$} & \multicolumn{3}{|c|}{$\begin{array}{l}\text { Período seco } \\
\text { Days dry }\end{array}$} \\
\hline & & $\begin{array}{l}\text { Quadrado médio } \\
\text { Mean square }\end{array}$ & $\begin{array}{l}\text { Niv. sign. } \\
\text { Sign. level }\end{array}$ & $\mathrm{R}^{2}$ & $\begin{array}{l}\text { Quadrado médio } \\
\text { Mean square }\end{array}$ & $\begin{array}{l}\text { Niv. sign. } \\
\text { Sign. level }\end{array}$ & $\mathrm{R}^{2}$ \\
\hline $\begin{array}{l}\text { Touro } \\
\text { Bull }\end{array}$ & 358 & $4.027,0$ & 0,0003 & 0,0854 & $2.543,19$ & 0,0691 & 0,0912 \\
\hline $\begin{array}{l}\text { Núcleo } \\
\text { Nucleous }\end{array}$ & 5 & $12.845,6$ & 0,0010 & 0,0038 & $13.959,13$ & 0,0001 & 0,0069 \\
\hline $\begin{array}{l}\text { Ano de parição } \\
\text { Calving year }\end{array}$ & 4 & $131.722,9$ & 0,0001 & 0,0312 & $59.014,47$ & 0,0001 & 0,0236 \\
\hline $\begin{array}{l}\text { Estação de parição } \\
\text { Calving season }\end{array}$ & 1 & $191.306,1$ & 0,0001 & 0,0113 & $34.879,39$ & 0,0001 & 0,0035 \\
\hline $\begin{array}{l}\text { Grupo genético (GG) } \\
\text { Genetic group }\end{array}$ & 2 & $1.762,5$ & 0,5687 & 0,0002 & $4.747,88$ & 0,1244 & 0,0009 \\
\hline $\begin{array}{l}\text { Nível de produção (NP) } \\
\text { Production level }\end{array}$ & 2 & $221.761,3$ & 0,0001 & 0,0263 & $171.090,45$ & 0,0001 & 0,0343 \\
\hline $\begin{array}{l}\text { Idade em classes (IDC) } \\
\text { Calving age classes }\end{array}$ & 14 & $2.024,8$ & 0,8259 & 0,0017 & $4.981,06$ & 0,0064 & 0,0069 \\
\hline $\begin{array}{l}\text { GGxNP } \\
\text { GGxIDC } \\
\text { NPx IDC } \\
\text { Resíduo } \\
\text { Error } \\
\end{array}$ & $\begin{array}{c}4 \\
28 \\
28 \\
6038\end{array}$ & $\begin{array}{l}6.505,9 \\
2.081,9 \\
3.914,4 \\
3.122,5\end{array}$ & $\begin{array}{l}0,0802 \\
0,9077 \\
0,1677\end{array}$ & $\begin{array}{l}0,0015 \\
0,0034 \\
0,0065\end{array}$ & $\begin{array}{l}7.808,84 \\
1.553,51 \\
2.087,41 \\
2.277,20\end{array}$ & $\begin{array}{l}0,0083 \\
0,8946 \\
0,5913\end{array}$ & $\begin{array}{l}0,0031 \\
0,0043 \\
0,0058\end{array}$ \\
\hline $\begin{array}{l}\text { Coeficiente de variação }(\%) \\
\text { Coefficient of variation }(\%)\end{array}$ & & & 46,10 & & & 51,71 & \\
\hline
\end{tabular}


Rev. bras. zootec.

Tabela 2 - Número de observações (N), médias ajustadas dos períodos de serviço (PSER) e seco (PS) e seus respectivos erros-padrão (EP), de vacas da raça Holandês, de acordo com o núcleo

Table 2 - Number of observations ( $N$ ), adjusted means of days open (DO) and days dry (DD) and standard error (SE), of Holstein breed cows, according to the nucleus

\begin{tabular}{|c|c|c|c|}
\hline $\begin{array}{l}\text { Núcleo } \\
\text { Nucleus }\end{array}$ & $\mathrm{N}$ & $\begin{array}{c}\mathrm{PSER}(\mathrm{EP}) \\
D O(S E)\end{array}$ & $\begin{array}{l}\mathrm{PS}(\mathrm{EP}) \\
D D(S E)\end{array}$ \\
\hline N. C. G. H. de Juiz de Fora & 2675 & $129,39 \quad(2,90)$ & $99,65 \quad(2,48)$ \\
\hline N. C. G. H. do Alto do Rio Grande & 1518 & $128,57 \quad(3,05)$ & $94,19 \quad(2.60)$ \\
\hline A. C. G. H. do Sul e Sudoeste Mineiro & 1582 & $119,54 \quad(3,13)$ & $101,91 \quad(2,67)$ \\
\hline N. G. H. de Barbacena & 355 & $128,09 \quad(4,61)$ & $86,45 \quad(3,93)$ \\
\hline A. C. de Bovinos do Alto Paranaíba & 334 & $130,39 \quad(4,27)$ & $93,48 \quad(3,65)$ \\
\hline N. C. G. H. do Triângulo Mineiro & 21 & $121,66(13,53)$ & $86,40 \quad(11,55)$ \\
\hline
\end{tabular}

Tabela 3 - Número de observações $(\mathrm{N})$, médias ajustadas dos períodos de serviço (PSER) e seco (PS) e seus respectivos erro-padrão (EP), de vacas da raça Holandês, de acordo com o ano de parição Table 3 - Number of observations $(N)$, adjusted means of days open (DO) and days dry (DD) and standard error (SE), of Holstein cows, according to the calving year

\begin{tabular}{lccc}
\hline $\begin{array}{l}\text { Ano de parição } \\
\text { Calving year }\end{array}$ & $\mathrm{N}$ & $\begin{array}{c}\text { PSER }(\mathrm{EP}) \\
D O(S E)\end{array}$ & $\begin{array}{c}\text { PS (EP) } \\
\text { DD (SE) }\end{array}$ \\
\hline 1990 & 760 & $134,04(4,41)$ & $94,45(3,77)$ \\
1991 & 1242 & $128,76(4,07)$ & $92,46(3,47)$ \\
1992 & 1568 & $134,44(3,85)$ & $100,26(3,29)$ \\
1993 & 1951 & $131,05(3,65)$ & $99,90(3,11)$ \\
1994 & 964 & $103,07(3,67)$ & $81,35(3,14)$ \\
\hline
\end{tabular}

a primeira variável e de oscilações climáticas entre os anos, alterando a quantidade e qualidade dos alimentos, bem como a interferência do produtor no caso do PS. A variabilidade observada nos anos intermediários pode ser conseqüência da vulnerabilidade do setor leiteiro às políticas econômicas, levando o criador a intervir no manejo, atrasando a cobrição da vaca.

Os valores obtidos para PSER e PS, em 1994, apresentam-se mais próximos daqueles preconizados pela maior parte das pesquisas para se obter a máxima produção, que, em ambos os casos, deveriam se situar em torno de 60 dias (KHATTAB e ASHMAWY, 1988).

Vacas que pariram na estação da seca apresentaram PSER e PS médios menores que as paridas na estação das águas (Tabela 4). A maior incidência de partos na estação seca $(56,4 \%)$ sugere melhor condição corporal da vaca conseguida no final da estação das águas, aliada às condições climáticas mais amenas da estação seca, favorecendo a concepção, e também às melhorias no manejo nutricional do rebanho que vêm ocorrendo atualmente durante a estação seca. RIBAS et al. (1995) não verificaram efeito
Tabela 4 - Número de observações (N), médias ajustadas dos períodos de serviço (PSER) e seco (PS) e seus respectivos erro-padrão (EP), de vacas da raça Holandês, de acordo com a estação de parição ${ }^{1}$

Table 4 - Number of observations $(N)$, adjusted means of days open (DO) and days dry (DD) and standard error (SE). of Holstein cows, according to the calving season ${ }^{1}$

$\begin{array}{lll}\text { Ano de parição } \quad \mathrm{N} & \mathrm{PSER}(\mathrm{EP}) & \mathrm{PS}(\mathrm{EP})\end{array}$

\begin{tabular}{lccc} 
Calving year & & $D O(S E)$ & DD $(\mathrm{SE})$ \\
\hline Águas $($ Wet $)$ & 2829 & $132,08(3,68)^{\mathrm{a}}$ & $96,16(3,15)^{\mathrm{a}}$
\end{tabular}

Seca (Dry) $\quad 3656 \quad 120,46(3,58)^{\mathrm{b}} \quad 91,20(3,06)^{\mathrm{b}}$

${ }^{1}$ Médias seguidas de letras diferentes, na coluna, diferem pelo teste $\mathrm{F}(\mathrm{P}<0,01)$.

${ }^{1}$ Means, within a column, followed by different letters, are different by $F$ test $(P<.01)$

significativo para PSER. Redução no PS na estação da seca pode ser conseqüência de deliberado atraso na cobrição, visando à maior produção de leite no estádio inicial da lactação e, com isto, aumento ou manutenção da cota de leite. Isto ocasiona alongamento no período de lactação, com conseqüente diminuição no PS.

Para os níveis médio e alto de produção, não houve diferenças $(\mathrm{P}>0,05)$ quanto aos PSER e PS entre os grupos genéticos (Tabela 5), entretanto, no nível baixo, vacas 31/32 Holandês-Zebu apresentaram menor PSER $(\mathrm{P}<0,05)$, o que pode se justificar pela maior adaptação destes animais, na maioria das vezes, às condições adversas proporcionadas por ambientes tropicais. No caso do PS, os menores resultados foram também observados para vacas $31 / 32$ de baixo nível de produção $(\mathrm{P}<0,01)$.

Para qualquer um dos grupos genéticos, constatou-se maior PSER para as vacas de alta produção $(\mathrm{P}<0,01)$ em relação às demais, constatando-se, de modo geral, antagonismo entre nível de produção de leite e PSER. Com relação ao PS, vacas de alta produção apresentaram menores períodos secos. In- 
Tabela 5 - Médias ajustadas do período de serviço e seco, e seus respectivos erros-padrão, de vacas da raça Holandês, de acordo com o desdobramento da interação grupo genético e nível de produção*

Table 5 - $\quad$ Adjusted means of days open and days dry, and standard error of Holstein cows, according to the interaction of genetic group and production level

\begin{tabular}{|c|c|c|c|c|}
\hline \multirow[b]{2}{*}{$\begin{array}{l}\text { Grupo genético } \\
\text { Genetic group }^{1}\end{array}$} & \multicolumn{3}{|c|}{$\begin{array}{l}\text { Nível de produção } \\
\text { Production level }\end{array}$} & \multirow[b]{2}{*}{$\begin{array}{l}\text { Médias } \\
\text { Means }\end{array}$} \\
\hline & $\begin{array}{l}\text { Baixo } \\
\text { Low }\end{array}$ & $\begin{array}{l}\text { Médio } \\
\text { Average }\end{array}$ & $\begin{array}{l}\text { Alto } \\
\text { High }\end{array}$ & \\
\hline \multicolumn{5}{|l|}{$\begin{array}{l}\text { Período de serviço } \\
\text { Days open }\end{array}$} \\
\hline $31 / 32$ & $94,14(5,96)^{\mathrm{Bc}}$ & $114,50(4,91)^{\mathrm{Ab}}$ & $160,72(7,70)^{\mathrm{Aa}}$ & $123,12(4,92)$ \\
\hline $\begin{array}{l}\mathrm{PC} \\
\mathrm{PO} \\
\text { Médias } \\
\text { Means }\end{array}$ & $\begin{array}{l}105,67(5,20)^{\mathrm{Ac}} \\
112,00(7,02)^{\mathrm{Ab}} \\
103,94(4,84)\end{array}$ & $\begin{array}{l}118,22(3,48)^{\mathrm{Ab}} \\
121,00(4,93)^{\mathrm{Ab}} \\
117,91(3,46)\end{array}$ & $\begin{array}{l}155,97(4,77)^{\mathrm{Aa}} \\
154,22(5,97)^{\mathrm{Aa}} \\
156,97(4,57)\end{array}$ & $\begin{array}{l}126,62(3,61) \\
129,08(5,10)\end{array}$ \\
\hline \multicolumn{5}{|l|}{$\begin{array}{l}\text { Período seco } \\
\text { Days dry }\end{array}$} \\
\hline $\begin{array}{l}31 / 32 \\
\mathrm{PC} \\
\mathrm{PO} \\
\text { Médias } \\
\text { Means }\end{array}$ & $\begin{array}{l}112,63(5,09)^{\mathrm{Ba}} \\
130,20(4,44)^{\mathrm{Aa}} \\
124,61(5,99)^{\mathrm{Aa}} \\
122,48(4,12)\end{array}$ & $\begin{array}{l}81,19(4,20)^{\mathrm{Ab}} \\
83,18(2,97)^{\mathrm{Ab}} \\
84,87(4,21)^{\mathrm{Ab}} \\
83,08(2,96)\end{array}$ & $\begin{array}{l}72,74(6,58)^{\mathrm{Ab}} \\
77,19(4,07)^{\mathrm{Ab}} \\
76,52(5,10)^{\mathrm{Ac}} \\
75,48(3,91)\end{array}$ & $\begin{array}{l}88,85(4,20) \\
96,86(3,08) \\
95,33(4,36)\end{array}$ \\
\hline
\end{tabular}

fluências do grupo genético também foram verificadas por RIBAS et al. (1995), no Paraná.

A relação antagônica entre nível de produção e PSER pode ser explicada, fisiologicamente, pelo fato de a seleção para alta produção aumentar as concentrações sangüíneas de somatotropina e prolactina, em detrimento às concentrações de insulina, que é um hormônio antagônico à lactação e importante para o desenvolvimento folicular normal (NEBEL e McGILLIARD et al., 1993). Com isto, estas mudanças hormonais favorecem a alta produção de leite e se tornam potencialmente prejudiciais para as outras funções fisiológicas, no caso de o manejo nutricional não ser adequado para suprir as demandas metabólicas da lactação. Resultados semelhantes, em que vacas de alta produção têm PSER mais longo que as de baixa, foram obtidos por BAGNATO e OLTENACU (1994) e MARTI e FUNK (1994).

A semelhança entre os PS, para os animais 31/32 Holandês-Zebu e PC, com geração controlada nos níveis médio e alto de produção, pode ser em função de maior persistência da lactação, quando os animais apresentaram produções mais elevadas, diminuindo, com isso, o PS. Estes menores valores parecem estar relacionados às melhores condições de manejo nutricional e reprodutivo proporcionados. O PS médio encontrado para as vacas de alto nível de produção assemelha-se ao verificado por TEIXEIRA et al. (1995), o qual foi de 80 dias.

As classes de idade da vaca ao parto apresentaram uniformidade de PSER dos animais, ao longo de sua vida (Tabela 6), semelhante à tendência de uniformidade do PSER, com o avançar da idade da vaca, constatada por RIBAS et al. (1995). Entretanto, HILLERS et al. (1984) e MARTI e FUNK (1994) constataram que vacas mais velhas, em geral, apresentaram pior desempenho reprodutivo, com conseqüente aumento do PSER. Com relação ao PS, pela Figura 1, pode-se observar aumento com o avanço da idade, semelhante ao que foi observado por SCHAEFFER e HENDERSON (1972), os quais verificaram que as vacas mais velhas tenderam a apresentar PS anterior maior que as vacas jovens. Por outro lado, DIAS e ALLAIRE (1982) relataram que ocorre redução brusca no PS tido como ótimo para maximizar a produção, com o aumento do número de lactações ou idade da vaca, sendo que as vacas na quarta ordem de parto ou mais parecem requerer, aproximadamente, 27 dias, comparado aos 65 dias para as primíparas. 
Rev. bras. zootec.

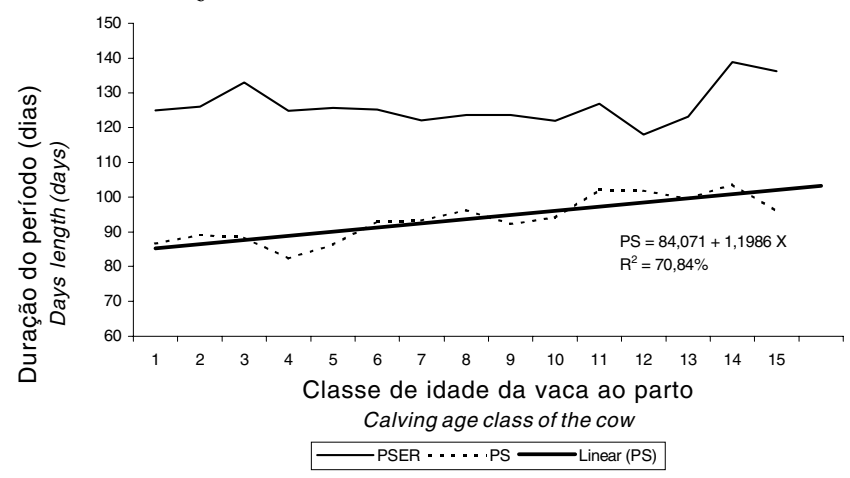

Figura 1 - Duração do período de serviço (PSER) e período seco (PS), de acordo com as classes de idade ao parto.

Figure 1 - Days open (PSER) and days dry (PS) length according to the calving ages classes.

\section{Parâmetros genéticos}

As estimativas das variâncias e covariâncias obtidas, respectivamente, nas análises uni e bivariada, sob modelo animal, são apresentadas na Tabela 7. As estimativas de herdabilidade $\left(\mathrm{h}^{2}\right)$ para PSER obtidas por análise univariada e bivariada $(0,052$ e 0,027 , respectivamente) são baixas, sendo semelhantes às obtidas por RAHEJA et al. (1989), HAYES et al. (1992), SILVA et al. (1992) e MOORE et al. (1992) e, também, por MARTI e FUNK (1994) e VALENTE et al. (1995), pelo método DFREML. As estimativas para PSER sugerem que a variância genética aditiva para esta característica é pequena, indicando que a sua expressão pode ser mais influenciada pela atuação de genes dominantes ou epistáticos ou, ainda, de fatores fisiológicos e de ambiente.

As estimativas de $\mathrm{h}^{2}$ para PS obtidas por análise uni e bivariada $(0,004$ e 0,0001 , respectivamente) foram extremamente baixas, sendo inferiores às obtidas por VALENTE et al. (1995), pelo mesmo método de estimação, porém com algumas diferenças quanto aos efeitos fixos considerados, sendo a principal delas a inclusão por esses autores da produção de leite total na lactação como covariável, obtendo $\mathrm{h}^{2}$ de 0,08 na presença da covariável e de 0,12 na ausência.

A maior participação da variância do efeito permanente de vaca e de ambiente temporário, em relação à variância aditiva, levou a estimativa de $\mathrm{h}^{2}$ do PS a praticamente igual a zero (Tabela 7). Isto leva a concluir que esta variável é predominantemente de efeito ambiental, respondendo mais a melhorias no manejo como um todo. Entretanto, na
1011

literatura, esta característica tem apresentado pequena fração herdável (não quase nula como a obtida neste trabalho), mesmo quando se considera o efeito permanente do animal.

As estimativas de repetibilidade para o PSER, pelas análises uni e bivariadas, foram de 0,06 Valores superiores haviam sido obtidos por SILVA et al. (1992). Estas estimativas inferiores devemse à baixa variância genética aditiva e do efeito permanente do animal. Esta baixa estimativa pode ser causada pelo fato destas características envolverem decisões pessoais dos produtores de leite, como momento de iniciar as coberturas pós-parto e definição do número máximo de serviços por concepção, adicionando-se a isto as dificuldades na detecção de cio e vários outros fatores de manejo e nutricional.

Para o PS, obtiveram-se estimativas de repetibilidade nas análises uni e bivariadas iguais a 0,03 . Este valor indica que não é aconselhável fazer predições de PS para lactações futuras, devido à pequena variância genética e de efeito permanente de ambiente existentes (Tabela 7). Portanto, melhorias para esta característica são mais fáceis de se obter com o aprimoramento das técnicas de manejo do que pela seleção.

Comumente, PSER e PS apresentam pequeno componente genético, o que pode ser evidenciado pelas baixas estimativas de $\mathrm{h}^{2}$ encontradas neste trabalho e citadas por diversos autores (SCHAEFFER e HENDERSON, 1972; SEYKORA e MAcDANIEL, 1983; MARTI e FUNK, 1994; e MAKUZA e McDANIEL, 1996). As correlações genética, fenotípica e de ambiente foram 0,55 ; 0,72 ; e 0,73 , respectivamente, sendo relativamente altas e positivas, indicando dependência destas características. Dessa forma, pode-se dizer que o PSER, possivelmente, apresenta relação biológica com PS, devido à razoável correlação genética positiva apresentada $(0,55)$, o que pode estar indicando que as vacas com piores desempenhos reprodutivos (PSER) têm maiores PS.

$\mathrm{Na}$ literatura, as estimativas quanto à correlação genética entre estas características mostram-se contraditórias. A exemplo disto, podem ser citadas as relatadas por MOORE et al. (1990), os quais obtiveram estimativas de 0,44 e 0,48 para as raças Ayrshire e Holandesa, respectivamente, enquanto MAKUZA e McDANIEL (1996), considerando também o efeito permanente de vaca, encontraram correlações de $-0,15$ e 0,54 na segunda e terceira lactações, respectivamente. 
PEREIRA et al.

Tabela 6 - Número de observações (N), médias ajustadas dos períodos de serviço (PSER) e seco (PS) e seus respectivos erros-padrão (EP) de vacas da raça Holandês, de acordo com a classe de idade da vaca ao parto

Table 6 - Number of observations (N), adjusted means of days open (DO) and days dry (DD) and standard errors (SE), of Holstein breed cows, according to the calving age class

\begin{tabular}{lcccr}
\hline $\begin{array}{l}\text { Classes } \\
\text { Class }\end{array}$ & $\begin{array}{c}\text { Classe de idade (meses) } \\
\text { Class of age (months) }\end{array}$ & $\mathrm{N}$ & $\begin{array}{c}\text { PSER (EP) } \\
D O(S E)\end{array}$ & $\begin{array}{r}\text { PS (EP) } \\
D D(S E)\end{array}$ \\
\hline 1 & $20-25$ & 268 & $124,9(5,9)$ & $86,7(5,0)$ \\
2 & $26-27$ & 576 & $126,1(4,6)$ & $89,1(3,9)$ \\
3 & $28-29$ & 682 & $132,9(4,6)$ & $88,4(3,9)$ \\
4 & $30-31$ & 724 & $124,8(4,3)$ & $82,4(3,7)$ \\
5 & $32-35$ & 954 & $125,7(3,9)$ & $86,3(3,3)$ \\
6 & $36-39$ & 618 & $125,2(4,0)$ & $92,9(3,4)$ \\
7 & $40-43$ & 740 & $122,0(3,9)$ & $93,2(3,4)$ \\
8 & $44-47$ & 550 & $123,6(4,2)$ & $96,2(3,6)$ \\
9 & $48-52$ & 425 & $123,6(4,8)$ & $92,3(4,1)$ \\
10 & $53-57$ & 368 & $121,9(5,1)$ & $94,1(4,3)$ \\
11 & $58-62$ & 225 & $126,9(5,9)$ & $102,1(5,1)$ \\
12 & $63-68$ & 181 & $118,0(7,1)$ & $101,9(6,1)$ \\
13 & $69-74$ & 107 & $123,2(8,5)$ & $99,6(7,3)$ \\
14 & $75-82$ & 47 & $138,8(15,5)$ & $103,7(13,2)$ \\
15 & $\geq 83$ & 20 & $136,2(20,8)$ & $96,0(17,8)$ \\
\hline
\end{tabular}

Tabela 7 - Variâncias e covariâncias genéticas do efeito permanente de ambiente (EPA) e do efeito temporário de ambiente (ETA) e fenotípicas das análises univariada e bivariada para períodos de serviço (PSER) e seco (PS)

Table 7 - Genetic, permanent environmental effect (PEE), temporary environmental effect (TEE) and phenotypic variances and covariance of univariate and bivariate analysis for days open (DO) and days dry $(D D)$

\begin{tabular}{|c|c|c|}
\hline $\begin{array}{l}\text { Variável } \\
\text { Trait }\end{array}$ & $\begin{array}{c}\text { Período de serviço } \\
\text { Days open }\end{array}$ & $\begin{array}{c}\text { Período seco } \\
\text { Days dry }\end{array}$ \\
\hline \multicolumn{3}{|l|}{ Análise univariada } \\
\hline \multicolumn{3}{|l|}{ Univariate analysis } \\
\hline Variância genética & 154,6730 & 0,7705 \\
\hline \multicolumn{3}{|l|}{ Genetic variance } \\
\hline Variância efeito permanente de ambiente & 41,7779 & 69,1873 \\
\hline \multicolumn{3}{|l|}{ Permanent environmental effect variance } \\
\hline Variância efeito temporário de ambiente & 2785,7615 & 2034,4261 \\
\hline \multicolumn{3}{|l|}{ Environmental variance } \\
\hline Variância fenotípica & 2982,2124 & 2104,3838 \\
\hline \multicolumn{3}{|l|}{ Phenotypic variance } \\
\hline \multicolumn{3}{|l|}{ Análise bivariada } \\
\hline \multicolumn{3}{|l|}{ Bivariate analysis } \\
\hline PSER (variância genética) & 81,8075 & $(2,2249)$ \\
\hline \multicolumn{3}{|l|}{ DO (genetic variance) } \\
\hline PS (variância genética) & - & 0,2015 \\
\hline \multicolumn{3}{|l|}{$D D($ genetic variance $)$} \\
\hline PSER (variância EPA) & 106,0790 & $(69,5594)$ \\
\hline \multicolumn{3}{|l|}{$D O(P E E$ variance $)$} \\
\hline PS (variância EPA) & - & 72,6787 \\
\hline \multicolumn{3}{|l|}{$D D(P E E$ variance $)$} \\
\hline PSER (variância ETA) & 2789,1623 & $(1739,3274)$ \\
\hline \multicolumn{3}{|l|}{ DO (TEE variance) } \\
\hline PS (variância ETA) & - & 2031,2534 \\
\hline \multicolumn{3}{|l|}{$D D(T E E$ variance $)$} \\
\hline PSER (variância fenotípica) & 2977,0488 & $(1811,1117)$ \\
\hline \multicolumn{3}{|l|}{ DO (phenotypic variance) } \\
\hline PS (variância fenotípica) & - & 2104,1336 \\
\hline$D D$ (phenotypic variance) & & \\
\hline
\end{tabular}




\section{Conclusões}

Existem vários fatores que devem ser corrigidos para que PSER e PS se situem no nível ideal de em torno dos 60 dias.

Os períodos de serviço e seco possuem herdabilidade e repetibilidade muito baixas.

Em estudos de características que envolvem o período seco, estas devem ser ajustadas para este fator, por ser o mesmo de efeito amplamente ambiental.

Vacas com maior período de serviço apresentam maiores períodos secos.

\section{Agradecimento}

À Associação dos Criadores de Gado Holandês de Minas Gerais, pela concessão dos dados para a realização deste trabalho.

\section{Referências Bibliográficas}

BAGNATO, A., OLTENACU, P.A. 1994. Phenotypic evaluation of fertility trais and their association with milk production of Italian Friesian cattle. J. Dairy Sci., 77(3):874-882.

BOLDMAN, K.G., KRIESE, L.A., Van VLECK, L.D. et al. 1995. A manual for use of MTDFREML. A set of Programs to obtain estimates of variances and covariance's (DRAFT). U.S. Department of Agriculture, Agricultural Research Service. $115 \mathrm{p}$.

BUTTE, S.V., DESHPANDE, K.S. 1987. Studies on dry period and service period in Friesian $\mathrm{x}$ Sahiwal cross-breds. Ind. Vet. $J ., 64(2): 152-155$.

CAMPOS, M.S., WILCOX, C.J., BECERRIL, C.M. et al. 1994. Genetic parameters for yield and reproductive traits of Holstein and Jersey in Florida. J. Dairy Sci., 77(3):867-873.

CASTRO, J.O. de, NEIVA, R.S., OLIVEIRA, A.I.G. et al. Avaliação da eficiência reprodutiva de rebanhos da raça holandês no Estado de Minas Gerais. In: REUNIÃO ANUAL DA SOCIEDADE BRASILEIRA DE ZOOTECNIA, 32, Brasília, 1995. Anais... Brasília: SBZ, 1995, p.453-455.

DIAS, F.M., ALLAIRE, F.R. 1982. Dry period to maximize milk production over two consecutive lactations. J. Dairy Sci., 65(1):136-145.

DIAS, J.P., FONSECA, F.A., TORRES, C.A.A. et al. 1985. Influência de fatores de meio ambiente e manejo sobre a eficiência reprodutiva do rebanho de vacas puras e mestiças holandesas da UFV. R. Soc. Bras. Zootec., 14(1):119-129.

FUNK, D.A., FREEMAN, A.E., BERGER, P.J. 1987. Effects of previous days open, previous days dry, and present days open on lactation yield. J. Dairy Sci., 70(11):2366-2373.

HAYES, J.F., CUE, R.I., MONARDES, H.G. 1992. Estimates of repeatability of reproductive measures in Canadian Holstein. J. Dairy Sci., 75(6):1701-1706.

HILLERS, J.K., SENGER, P.L., DARLINGTON, R.L. et al. 1984. Effects of production, season, age of cows, days dry, and days in milk on conception to first service in large commercial dairy herds. J. Dairy Sci., 67(4):861-867.

KHATTAB, A.S., ASHMAWY, A.A. 1988. Relationships of days open and days dry with production in Friesian cattle Egypt. J. Anim. Breed. Gen., 105: 300-305.

MAKUZA, S.M., McDANIEL, B.T. 1996. Effects of days dry, previous days open, and current days open on yields of cows in Zimbabwe and North Carolina. J. Dairy Sci., 79(4):702-709.

MARTI, C.F., FUNK, D.A. 1994. Relationship between production and days open at different levels of herd production. J. Dairy Sci., 77(6):1682-1690.

MOORE, R.K., KENNEDY, B.W., SCHAEFFER, L.R. et al. 1990. Relationships between reproduction traits, age and body weight at calving, and days dry in first lactation Ayrshires and Holsteins. J. Dairy Sci., 73(3):835-842.

MOORE, R.K., KENNEDY, B.W., SCHAEFFER, L.R. et al. 1972. Relationships between age and body weight at calving, feed intake, production, days open, and selection indexes in Ayrshires and Holsteins. J. Dairy Sci., 75(1):294-306.

NEBEL, R.L., McGILLIARD, M.L. 1993. Interactions of high milk yield and reproductive performance in dairy cows. J. Dairy Sci., 76(10):3257-3268.

RAHEJA, K.L., BURNSIDE, E.B., SCHAEFFER, L.R. 1989. Relationships between fertility and production in Holstein dairy cattle in different lactation's. J. Dairy Sci., 72(10):2670-2678.

RIBAS, N.P., MILAGRES, J.C., CASTRO, A.C.G. et al. 1984. Influência do intervalo de partos e do período seco sobre a produção de leite, gordura e procentagem de gordura em rebanhos holandeses da bacia de Castrolândia, Estado do Paraná. R. Soc. Bras. Zootec., 13(2):225-234.

RIBAS, N.P., MONARDES, H.G., RICHTER, G.O. et al. Estudo do intervalo entre partos e período de serviço em vacas da raça Holandês, na região de Witmarsum, Paraná. In: REUNIÃO ANUAL DA SOCIEDADE BRASILEIRA DE ZOOTECNIA, 32, Brasília, 1995. Anais... Brasília: Sociedade Brasileira de Zootecnia, 1995, p.697-698.

STATISTICAL ANALYSIS SYSTEM - SAS - 1996. User's Guide: Statistics, Cary: SAS INSTITUTE. 956p.

SCHAEFFER, L.R., HENDERSON, C.R. 1972. Effects of days dry and days open on Holstein milk production. J. Dairy Sci., 55(1):107-112.

SEYKORA, A.J., McDANIEL, B.T. 1983. Heritabilities and correlations of lactation yields and fertility for Holsteins. J. Dairy Sci., 66(7):1486-1493.

SILVA, H.M., WILCOX, C.J., THATCHER, W.W. et al. 1992. Factors affecting days open, gestation length, and calving interval in Florida dairy cattle. J. Dairy Sci., 75(1):288-293.

SILVA, M.V.G.B. da. Utilização de modelos animais uni e bivariados no estudo das relações entre eficiência reprodutiva e produção de leite na raça Holandês. Belo Horizonte, UFMG, 1995. 188p. Dissertação (Mestrado em Zootecnia) - Universidade Federal de Minas Gerais, 1995.

SMITH, J.W., LEGATES, J.E. 1962. Relation of days open and days dry to lactation milk and fat yields. J. Dairy Sci., 45(10):1192-1197.

TEIXEIRA, N.M., VALENTE, J., FREITAS, A.F. et al. Influência dos períodos de serviço e seco sobre a produção de leite em 305 dias na raça Holandês. In: REUNIÃO ANUAL DA SOCIEDADE BRASILEIRA DEZOOTECNIA, 32, Brasília, 1995. Anais... Brasília: SBZ, 1995, p.706-708.

VALENTE, J., TEIXEIRA, N.M., VERNEQUE, R.S. Efeitos de fatores genéticos e não genéticos sobre o período seco entre o primeiro e o segundo parto de vacas da raça Holandês. In: REUNIÃO ANUAL DA SOCIEDADE BRASILEIRA DE ZOOTECNIA, 31, Maringá, 1994. Anais... Maringá: SBZ, 1994, p.225.

VALENTE, J., TEIXEIRA, N.M., VERNEQUE, R.S. Efeitos dos períodos de serviço anterior, período seco anterior e período de serviço corrente sobre a produção de leite. In: REUNIÃO ANUAL DA SOCIEDADE BRASILEIRA DE ZOOTECNIA, 32, Brasília, 1995. Anais... Brasília: SBZ, 1995, p.686-688.

Recebido em: 18/06/1998

Aceito em: 30/05/2000 